\section{Endovascular laser treatment of mixed shunt}

\section{Alvise Cavallini \\ Private Practitioner, Verona, Italy}

\begin{abstract}
In this paper we present our endovascular LASER treatment of mixed shunts. This technique is built according the CHIVA's important principles: diastolic reflux is interrupts at its proximal origin by flush LASER obliteration while centripetal systolic reflux is preserved. In this way, the surgeon interrupts a recirculation pressure loop producing the ambulatory venous hypertension critical to the development of varicose vein symptoms. In this paper we explain how to better understand the intricate venous drainage pathophysiology of mixed shunt and the strategy to approach it using an office-based technique. With the correct strategy and the technological support it is possible to achieve a good functional and aesthetic result to prevent varicosity recurrence.
\end{abstract}

\section{Introduction}

If the varicose veins were only caused by diastolic reflux, phlebology would be easy matter; it would be enough to treat escape points (EP) and/or refluxes to solve or improve the hemodynamic pattern. The major problem is when a systolic reflux occurs. This type of reflux, more rare than diastolic one, can generate 2 types of shunt: an open by-passing shunts (OBS, also known as open vicarious shunts) or a mixed shunt, i.e. a combination of closed shunt (CS) and OBS.,2

In $\mathrm{CS}$, a vicious circle is created between the EP and the so-called re-entry point (RP). The deviated flow re-circulates at each energy gradient inversion, like in a close circuit. The energy gradient inversion is created every time a calf muscle systole is followed by the muscular relaxation (diastole), which means at every footstep.

An OBS is a natural bypass, exploited by the venous network to go over an obstacle. The use of a collateral route to by-pass a functional or a thrombotic occlusion is desirable, as it reduces the drainage resistance. For this reason, this type of shunts are not to be treated.

In mixed shunt (Figures 1 and 2), the two shunt types share the same EP and part of their refluxing pattern toward the corre- sponding different RP.

In this paper we present our officebased technique to treat mixed shunts with escape point originally from the popliteal fossa, using endovascular laser technology (EVLT).

In Figure 1 clinical case is schematized. The OBS presented a continuous flow, both is in systoles and diastole (total activation); sometime the activation may be only systolic, if the obstruction causes the opening of the shunt is not complete and therefore a flow rate is still allowed for the physiological route but, upon arrival of the systolic flow wave as a result of the activation of the pumps, drainage also requires an alternative route.

If the Giacomini's vein drains into an incompetent GSV and re-entry point is placed below the escape point, a centripetal flow also exist in the Giacomini's vein during muscle relaxation.

Blood enters the OBS for need of drainage, reaching the GSV reflux in diastole in the CS causing a mixed shunt; this pattern may cause signs and symptoms of chronic venous disease, like in our case.

Figure 2 shows our patient. A musculotendinous band called the vastoadductor membrane, which jointed the adductor tendon to the vastus medialis, can create a notch with a venous stenosis at the outlet of the Hunter's canal, usually located 12-14 $\mathrm{cm}$ above the femoral condyle. The vein at this level could be easily compressed in the posterior angle of the hiatus, commonly tightened by a calcified artery.

Figure 2A: the Giacomini's vein is ectatic and varicose, draining the blood during the muscular systole, in anterograde direction, coming into the GSV in the third distal thigh. Figure 2B and C: part of the blood continues to GSV in the anterograde direction reaching the femoral vein (OBS), another part overloads the GSV running backwards to the leg (CS), where the reflux moves to the clinically evident varicose tributary which causes hypodermitis and trophic ulceration.

\section{Case Report}

The popliteal fossa may present various pathological drainage patterns that need to be known to avoid the formation of surgically or sclerotherapic unviable cavernomas if it is an expression of a demolition of OBS. It is to be considered that the hemodynamics of the popliteal region differs from the sapheno-femoral one for the particular proximity of this area with one of the primary pumps among all the series pumps we
Correspondence: Alvise Cavallini, Private Practitioner, Lungadige Cangrande 10, 37126 Verona, Italy.

Tel.: +39.338.6647913 - Fax: +39.0458341088 E-mail: alvise.cavallini@tiscali.it

Key words: Laser treatment; shunt; endovascular treatment.

Received for publication: 3 September 2017. Revision received: 10 September 2017. Accepted for publication: 12 September 2017.

This work is licensed under a Creative Commons Attribution 4.0 License (by-nc 4.0).

(C) Copyright A. Cavallini, 2017

Licensee PAGEPress, Italy

Veins and Lymphatics 2017; 6:7048

doi:10.4081/vl.2017.7048

find in the lower limbs: the calf pump. In fact, there are high speed systoles in this area and strong energies are created in the OBS in the presence of organic or functional obstacles to popliteal-femoral physiological drainage.

The case we present (Figure 2) is a mixed shunt and the OBS is caused by Hunter's outlet syndrome; ${ }^{3}$ the patient in fact did not report episodes of deep venous thrombosis (DVT) and no evidence of DVT at Doppler-ultrasound (DUS) examination were highlighted. Compression of the femoral vein inside the adductor's canal is an underestimated and misdiagnosed cause of postural stenosis of the femoral vein. Typically, in these cases, the Giacomini's vein becomes the EP for the OBS; the venous blood shunts in a para-physiological pattern aimed to preserve the same limb drainage.

In this case, the Giacomini's vein allows an inverted flow (from the deepest popliteal vein - toward the more superficial compartment), so overloading the saphenous trunk while by-passing the obstacle (Figure 1). The anomalous pathway re-enter into femoral vein by means of the saphenofemoral junction that, in this example, becomes the RP. The terminal valve and the proximal portion of the GSV were competent both with sqeezing and with the Valsalva maneuvers. ${ }^{4}$

In some cases, like the present one, part of the blood below the re-entry of the Giacomini's vein into the GSV flow also in retrograde manner, so dilating the great saphenous vein (GSV) and making it refluxing. This refluxing pattern, constituting a vicious re-circulation, can cause signs and symptoms of chronic venous disease (CVD).

Because after the digit compression of 
the leg varicose tributary (reflux elimination test $)^{4}$ the reflux in the GSV was still present, our strategy was to perform a laser fragmentation of the hydrostatic column below the re-entry of the Giacomini into the GSV, while maintaining the upper outflow point of the OBS.

\section{Technique}

EVLT was performed with a $1470 \mathrm{~nm}$ diode laser (Ceralas E; Biolitec AG, Wien, Germany) and a commercial kit (ELVeS Radial $^{\mathrm{TM}} \mathrm{Kit} /$ Venflon ${ }^{\mathrm{TM}}$; Biolitec AG) containing all the equipment for the procedure (16-gauge needle for percutaneous introduction, $600 \mu \mathrm{c}$ double radial fiberoptic). EVLT was performed in an operating room with an ECG and pressure gauges monitoring. Only the short section of GSV with dyastolic reflux was treated (about $10 \mathrm{~cm}$ )
(Figure 3). GSV was accessed percutaneously with DUS guidance (Vivide, GE Healthcare, US). Using the 16-gauge introducer needle, the laser fiber tip has been positioned in close proximity to the Giacomini's vein confluence (Figure 3). Intraoperative DUS was used to guide the laser tip position and the delivery of tumescent anesthesia (cold $5^{\circ} \mathrm{C}$ saline solution $0.9 \%+1 \mathrm{fL}$ of lidocaine $2 \%+1 \mathrm{fL}$ of bicarbonate); this was infiltrated generously around the GSV by using a 25-gauge needle, creating a good halo effect and good compression of the vein. EVLT was carried out in a continuous mode with a power of 8 W. The pullback speed on the fiber was calculated to achieve a Linear Endovenous Energy Density (LEED: energy amount in joules divided by the treated vein length in centimeters) of $80 \mathrm{~J} / \mathrm{cm}$. After the procedure, venous outflow was checked immediately in the proximal GSV veins by DUS. In the same session all insufficient tributaries were treated by phlebectomy. Compression with 20-30 mmHg elastic stocking for two weeks was applied. In addition, as a precaution, low-molecular-weight heparin for six days was given at prophylactic dosage. Patient was mobilized immediately after the intervention and were advised to walk regularly during recovery from treatment.

We did not observe any particular complication or side effect. After 6 months patient was very satisfied with the method.

Figure 4 shows results at six months, with the disappearance of the leg varicose veins and the re-epithelialization of the ulcer. Giacomini's vein, although untreated, is less dilated, because the aspiration effect caused by vicious circulation has failed, so the flow rate is reduced.

As evident in Figure 3, the fiber tip was positioned 1-2 $\mathrm{cm}$ below the orifice of the Giacomini's vein under US guidance, also confirmed by direct visualization of the red aiming beam through the skin.

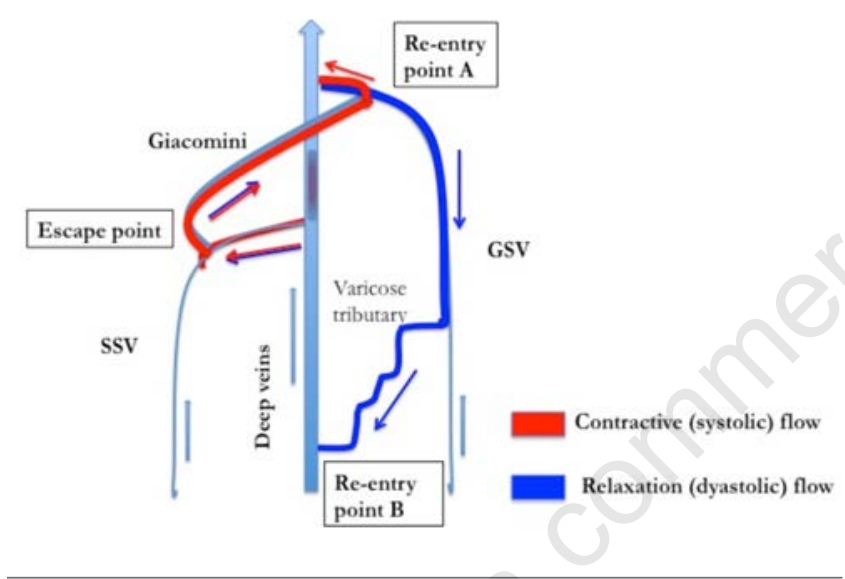

Figure 1. Mixed shunt.

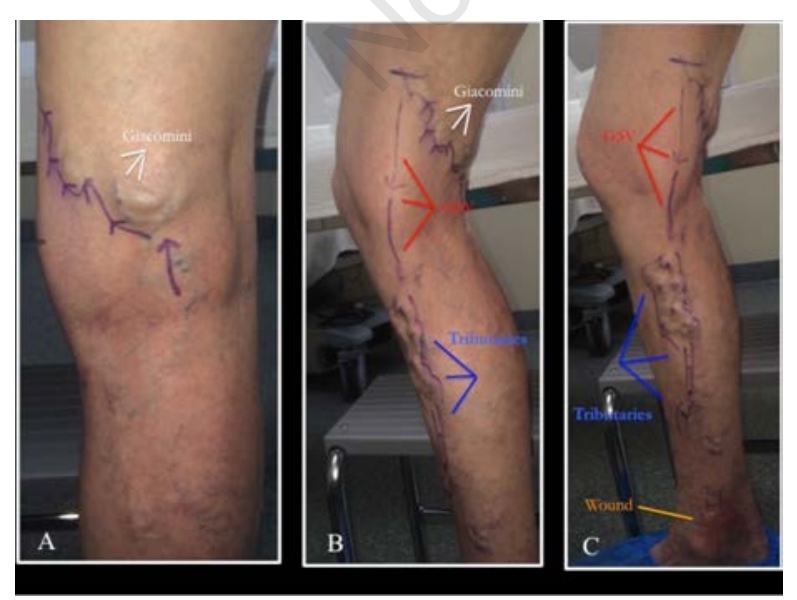

Figure 2. Patient 78 years old with mixed shunt which causes severe symptomatic chronic venous insufficiency with trophic ulceration $(\mathrm{C} 2,3,4,5,6 \mathrm{~s}$ pAs $+\mathrm{dPr}+\mathrm{o} 13,2,3,5$ according to CEAP classification).

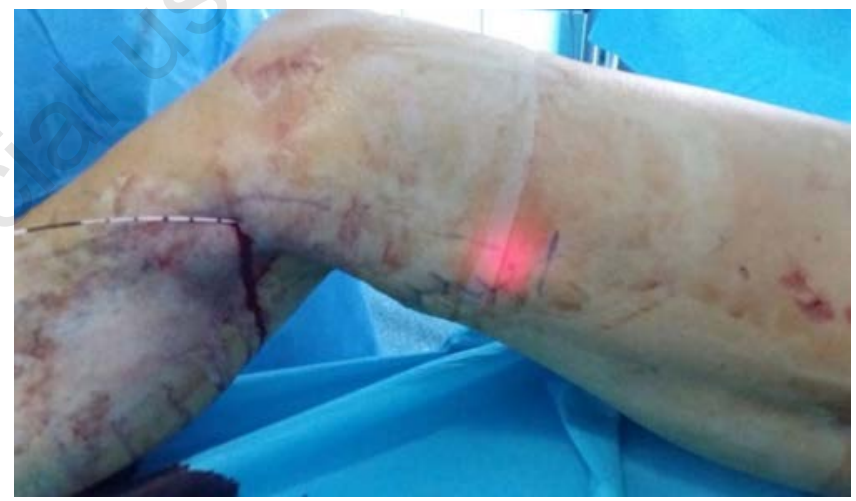

Figure 3. EVLT of refluxing GSV.

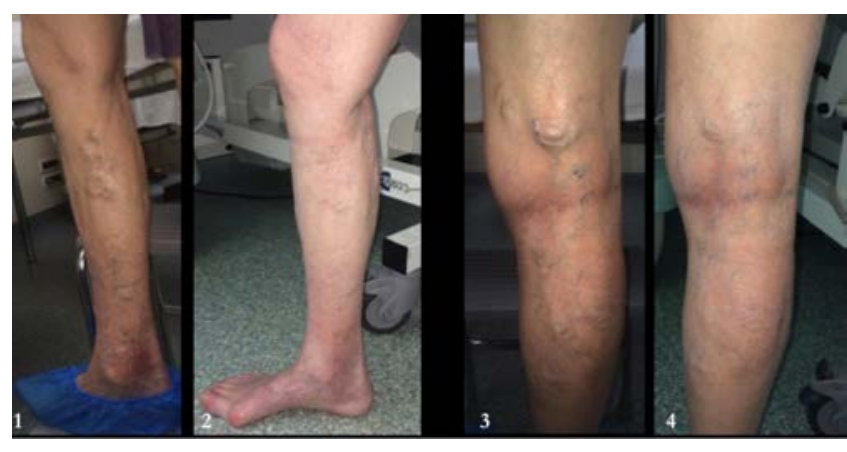

Figure 4. Medial and posterior aspect of leg and thigh; 1 and 3, before EVLT; 2 and 4, 6 months after EVLT. 


\section{Discussion and Conclusions}

In the last 15 years vein surgery has been largely replaced by percutaneous office-based procedures that can be performed under local or tumescent anesthesia with similar early and midterm results but with less discomfort to the patient, improved early QOL, and earlier return to work; saphenous vein thermal ablation is nowadays the gold standard according both American venous forum and the European and American Societies for Vascular Surgery. ${ }^{5,6}$

As in any field of medicine, however, the correct indication of treatment is crucial; not only technically but also strategically less aggressive approach could reduce the recurrence risk. Other Authors already reported EVLT according to CHIVA principles with good results. ${ }^{7,8}$

In our case, performing a standard laser procedure of the GSV would be useless (proximal GSV and terminal valve were competent) and especially harmful, because interrupting OBS would meet unavoidable varicose veins recurrence, very difficult to manage.

We consider this individualized EVLT strategy extremely useful in these complex shunts, because it allows: i) to preserve systolic centripetal reflux in Giacomini's vein; ii) to treat only diastolic vicious circulation without interrupting the centripetal flow; iii) to achieve a good functional and aesthetic result with a mini-invasive and endovascular method, with minimum discomfort to the patient and earlier return to work.

We conclude by quoting Glauco Bassi, an Italian phlebology master; He wrote in 1962:

The varicose treatment must be polynomial, etiologic, functional and, above all, differentiated, ie different from case to case. Under these conditions it provides excellent, lasting and predictable results with remarkable accuracy.

We continue to think the same way, technology today makes it easier.

\section{References}

1. Gianesini S, Occhionorelli S, Menegatti E, et al. CHIVA strategy in chronic venous disease treatment: instructions for users. Phlebology 2015;30:157-71.

2. Franceschi C, Zamboni P. Principles of venous hemodynamics. New York: Nova Biomedical Books; 2009.

3. Uhl JF, Gillot C. Anatomy of the Hunter's canal and its role in the venous outlet syndrome of the lower limb. Phlebology 2015;30:604-11.

4. Zamboni P, Gianesini S, Menegatti E, et al. Great saphenous varicose vein surgery without saphenofemoral junction disconnection. Br J Surg 2010;97:8205.

5. Gloviczki P, Comerota AJ, Dalsing MC, et al. The care of patients with varicose veins and associated chronic venous diseases: clinical practice guidelines of the Society for Vascular Surgery and the American Venous Forum. J Vasc Surg 2011;53:2S-48S.

6. Wittens C, Davies AH, Bækgaard N, et al. Editor's Choice - Management of Chronic Venous Disease: Clinical Practice Guidelines of the European Society for Vascular Surgery (ESVS). Eur J Vasc Endovasc Surg 2015;49:678737.

7. Gianesini S, Menegatti E, Zuolo M, et al. Short endovenous laser ablation of the great saphenous vein in a modified CHIVA strategy. Veins and Lymphatics 2013;2:e21.

8. Gianesini S, Menegatti E, Zuolo M, et al. Laser-assisted strategy for reflux abolition in a modified CHIVA approach. Veins and Lymphatics 2015;4:5246. 